\title{
Non-LTE Calculations of the Fe I $6173 \AA$ Line in a Flaring Atmosphere
}

\author{
Jie Hong ${ }^{1,2}$ (1) , M. D. Ding ${ }^{1,2}$ (1) ${\text {, Ying } \mathrm{Li}^{3} \text { (1), and Mats Carlsson }}^{4,5}$ (1) \\ ${ }^{1}$ School of Astronomy and Space Science, Nanjing University, Nanjing 210023, People’s Republic of China; dmd@ @ nju.edu.cn \\ ${ }^{2}$ Key Laboratory for Modern Astronomy and Astrophysics (Nanjing University), Ministry of Education, Nanjing 210023, People's Republic of China \\ ${ }^{3}$ Key Laboratory of Dark Matter and Space Astronomy, Purple Mountain Observatory, Chinese Academy of Sciences, Nanjing 210034, People's Republic of China \\ ${ }_{5}^{4}$ Rosseland Centre for Solar Physics, University of Oslo, P.O. Box 1029 Blindern, NO-0315 Oslo, Norway \\ ${ }^{5}$ Institute of Theoretical Astrophysics, University of Oslo, P.O. Box 1029 Blindern, NO-0315 Oslo, Norway \\ Received 2018 January 24; revised 2018 March 23; accepted 2018 March 26; published 2018 April 6
}

\begin{abstract}
The Fe I $6173 \AA$ A line is widely used in the measurements of vector magnetic fields by instruments including the Helioseismic and Magnetic Imager (HMI). We perform non-local thermodynamic equilibrium calculations of this line based on radiative hydrodynamic simulations in a flaring atmosphere. We employ both a quiet-Sun atmosphere and a penumbral atmosphere as the initial one in our simulations. We find that, in the quiet-Sun atmosphere, the line center is obviously enhanced during an intermediate flare. The enhanced emission is contributed from both radiative backwarming in the photosphere and particle beam heating in the lower chromosphere. A blue asymmetry of the line profile also appears due to an upward mass motion in the lower chromosphere. If we take a penumbral atmosphere as the initial atmosphere, the line has a more significant response to the flare heating, showing a central emission and an obvious asymmetry. The low spectral resolution of HMI would indicate some loss of information, but the enhancement and line asymmetry are still kept. By calculating polarized line profiles, we find that the Stokes $I$ and $V$ profiles can be altered as a result of flare heating. Thus the distortion of this line has a crucial influence on the magnetic field measured from this line, and one should be cautious in interpreting the magnetic transients observed frequently in solar flares.
\end{abstract}

Key words: line: profiles - radiative transfer - Sun: flares - Sun: photosphere

\section{Introduction}

The Fe I $6173 \AA$ line is thought to be formed in the photosphere, with a formation height of around $200-300 \mathrm{~km}$ (Norton et al. 2006; Bello González et al. 2009). This line is magnetically sensitive and has a good performance in vector magnetic field measurements (Norton et al. 2006). Thus, it has been selected for polarimetric observations in many solar instruments, including the Helioseismic and Magnetic Imager (HMI; Schou et al. 2012). In quiet regions and even regions with some activity, this line is mostly in absorption and quite stable. However, it is possible that this line can be affected by flares, in which the deeper layers are more or less heated. The change in the shape of the line profile has an influence on the inversions made to determine the magnetic field.

One example is the magnetic transients, the origins of which are still a subject of debate. They are observed as sudden changes in the magnetic field and even in the sign in some flares (Patterson \& Zirin 1981; Patterson 1984). Patterson (1984) argued that flare emissions can cause magnetic transients. Qiu \& Gary (2003) found that magnetic transients occur at the location of strong hard X-ray emissions. Many other studies also reported such magnetic transients that are not "real" but rather the results of line profiles altered during flares (Maurya \& Ambastha 2009; Maurya et al. 2012).

Previous studies of magnetic transients mainly employ data from the Michelson Doppler Imager (Scherrer et al. 1995), which uses the Ni I $6768 \AA$ line. Ding et al. (2002) carried out a detailed study of this line in a flaring atmosphere, and found that this line can go into emission when a relatively cool atmosphere (like the sunspot penumbra) is heated by an electron beam. Harker \& Pevtsov (2013) first used a semiempirical flare model to synthesize the Fe I $6173 \AA$ line profile observed by HMI. Sharykin et al. (2017) calculated the line profile from RADYN simulation results, and found that the intensity change is still smaller in magnitude compared with observations. Their calculations are only based on the quietSun atmosphere. However, many magnetic transients are observed in umbral and/or penumbral regions (Kosovichev \& Zharkova 2001; Burtseva et al. 2015). Therefore, we should also consider a cooler initial atmosphere, as in Ding et al. (2002).

There has been a large number of magnetic transient observations with HMI, yet the detailed process still needs more investigation, especially the response to flare heating. In this Letter, we investigate the response of the Fe I $6173 \AA$ line to flare heating using radiative hydrodynamic simulations for both a quiet-Sun atmosphere and a penumbral atmosphere. The remainder of this Letter is arranged as follows. In Section 2 we describe the basic method and model parameters. We present the results for the two different atmospheres in Section 3, followed by a summary and discussions in Section 4 .

\section{Method}

RADYN is a radiative hydrodynamics code (Carlsson \& Stein 1992, 1995, 1997, 2002) that can implicitly solve the hydrodynamic and radiative transfer equations together using an adaptive grid (Dorfi \& Drury 1987). It has been applied to flare simulations (Abbett \& Hawley 1999; Allred et al. $2005,2015)$ to calculate the atmospheric response to a beam of nonthermal electrons injected at the coronal loop top. The electron beam heating rate is obtained by solving the FokkerPlanck equation following McTiernan \& Petrosian (1990). Atoms that are important in the chromosphere are treated in non-LTE: a hydrogen atom with six levels including continuum, a Ca II atom with six levels including continuum, and a helium atom with nine levels including continuum. All 
Table 1

List of Parameters of Flare Simulation

\begin{tabular}{|c|c|c|c|c|c|}
\hline Label & $F\left(\mathrm{erg} \mathrm{cm}^{-2} \mathrm{~s}^{-1}\right)$ & Total Duration (s) & Spectral Index & $E_{c}(\mathrm{keV})$ & Initial Atmosphere \\
\hline$\overline{\mathrm{FQa}}$ & $5 \times 10^{10}$ & 20 & 3 & 25 & Quiet Sun \\
\hline $\mathrm{FQb}$ & $5 \times 10^{9}$ & 20 & 3 & 200 & Quiet Sun \\
\hline $\mathrm{FPa}$ & $5 \times 10^{10}$ & 20 & 3 & 25 & Penumbra \\
\hline
\end{tabular}

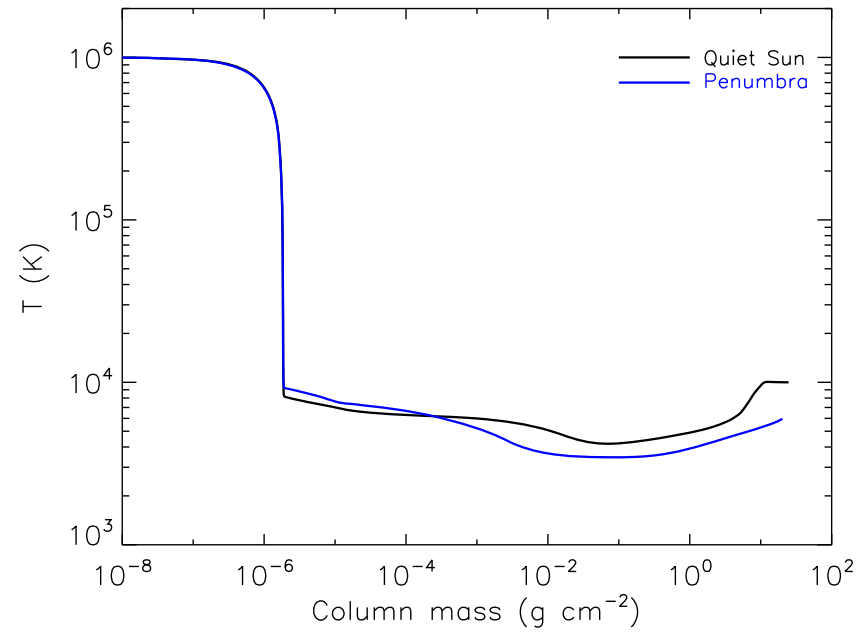

Figure 1. Distribution of temperature of the initial atmosphere for RADYN simulations after relaxation. The black curve is for the quiet Sun, and the blue curve is for the penumbra, respectively.

radiative transitions between the energy levels mentioned above are treated in complete frequency redistribution. Other atoms are treated in LTE with the background opacity package of Gustafsson (1973).

We assume an atmosphere in a $10 \mathrm{Mm}$ quarter-circular loop structure. Following Hong et al. (2017), we employ two different atmospheric models to construct the initial atmosphere. One is a quiet-Sun atmosphere, the same as in Hong et al. (2017), which is based on the VAL3C model (Vernazza et al. 1981); the other is a penumbral atmosphere that is based on the semi-empirical model of Ding \& Fang (1989). Figure 1 shows the two initial model atmospheres after relaxation, in which the penumbral model has a lower temperature in the lower atmosphere.

We assume that the initial atmosphere is subject to an electron beam heating during a flare. The electron beam is assumed to have a power-law distribution with a spectral index of 3 . The energy flux of the beam follows a triangular function over time, with a total duration of $20 \mathrm{~s}$. We consider two cases here: one with an average energy flux (half of the maximum; $F$ ) of $5 \times 10^{10} \mathrm{erg} \mathrm{cm}^{-2} \mathrm{~s}^{-1}$ and an ordinary low-energy cutoff $\left(E_{c}\right)$ of $25 \mathrm{keV}$; the other with a lower average energy flux of $5 \times 10^{9} \mathrm{erg} \mathrm{cm}^{-2} \mathrm{~s}^{-1}$ and a much higher low-energy cutoff of $200 \mathrm{keV}$. The former represents an intermediate flare. The latter mimics an extreme case like the white-light flare reported by $\mathrm{Xu}$ et al. (2006) in which the electrons can be accelerated to very high energies. We run both cases with the two different initial atmospheres, respectively. The model parameters of all four running cases are listed in Table 1.

Each case is run for $20 \mathrm{~s}$ and we save the simulation snapshots every $0.1 \mathrm{~s}$. The snapshots are then taken as input to the radiative transfer code RH (Uitenbroek 2001; Pereira \& Uitenbroek 2015) to calculate the Fe I $6173 \AA$ line profiles. For better convergence, when calculating this line we cut the atmosphere above $1.2 \mathrm{Mm}$ where the temperature is relatively high and the contribution to this line is negligible.

\section{Results}

\subsection{Quiet-Sun Atmosphere}

We show the evolution of the Fe I $6173 \AA$ line profile for all four cases in Figure 2. For cases FQa and FQb, we see a dimming in both the line and the nearby continuum during the first 2 s. The dimming effect caused by nonthermal electrons seems a common phenomenon in flares and Ellerman bombs, which has already been reported in some previous simulations (Abbett \& Hawley 1999; Allred et al. 2006; Hong et al. 2017). Dimming in this line is also seen in the calculation results of Sharykin et al. (2017). At the beginning, the line source function decouples from the local Planck function above the temperature minimum region, and the formation height of the line center (optical depth unity) is around $220 \mathrm{~km}$ (Figure 3). When flare heating sets in, the line formation height increases first, resulting in a dimming effect, and then decreases gradually when the upper atmosphere is heated. The decoupling between the line source function and the Planck function is also reduced because the nonthermal heating enhances the line source function. As a result, the contribution function to the line extends to a higher level in the atmosphere. At $10 \mathrm{~s}$ and $12 \mathrm{~s}$, the contribution function shows a small hump at around $550 \mathrm{~km}$ in addition to the main hump at the lower layers. An analysis of the energy budget in Case FQa shows that the electron beam heating is the dominant gain in the chromosphere (above $500 \mathrm{~km}$ ), while radiative heating (backwarming) is important in the photosphere where the line center is formed. These two energy terms contribute to a significant intensity increase at the line center, although the line profile remains in absorption. The line profile shows a very weak blue asymmetry due to an upward velocity $\left(\sim 1 \mathrm{~km} \mathrm{~s}^{-1}\right)$ in the lower chromosphere.

Case FQb adopts a very high cutoff energy of the electron beam, which is used to mimic a larger penetration depth of the beam electrons in some exceptionally energetic white-light flares (e.g., Xu et al. 2006). From Figure 3 we can see that direct heating by nonthermal electrons can indeed go into deeper layers than in FQa. Although the energy flux of the electron beam is one order smaller of magnitude in Case FQb, the increase at the line center has a similar magnitude to that in Case FQa. Naturally, in Case FQb, the contribution of electron beam heating to the line intensity is relatively larger than in Case FQa, as judged from the obvious hump in the contribution function at 10 and $12 \mathrm{~s}$. There is still a weak blue asymmetry in the line profile in Case FQb.

In order to compare the model results with real HMI observations, we then convolve our calculated line profiles with the six HMI transmission functions to obtain the simulated HMI profiles following Harker \& Pevtsov (2013). These results are superimposed to Figure 2 as diamonds. It is seen that 

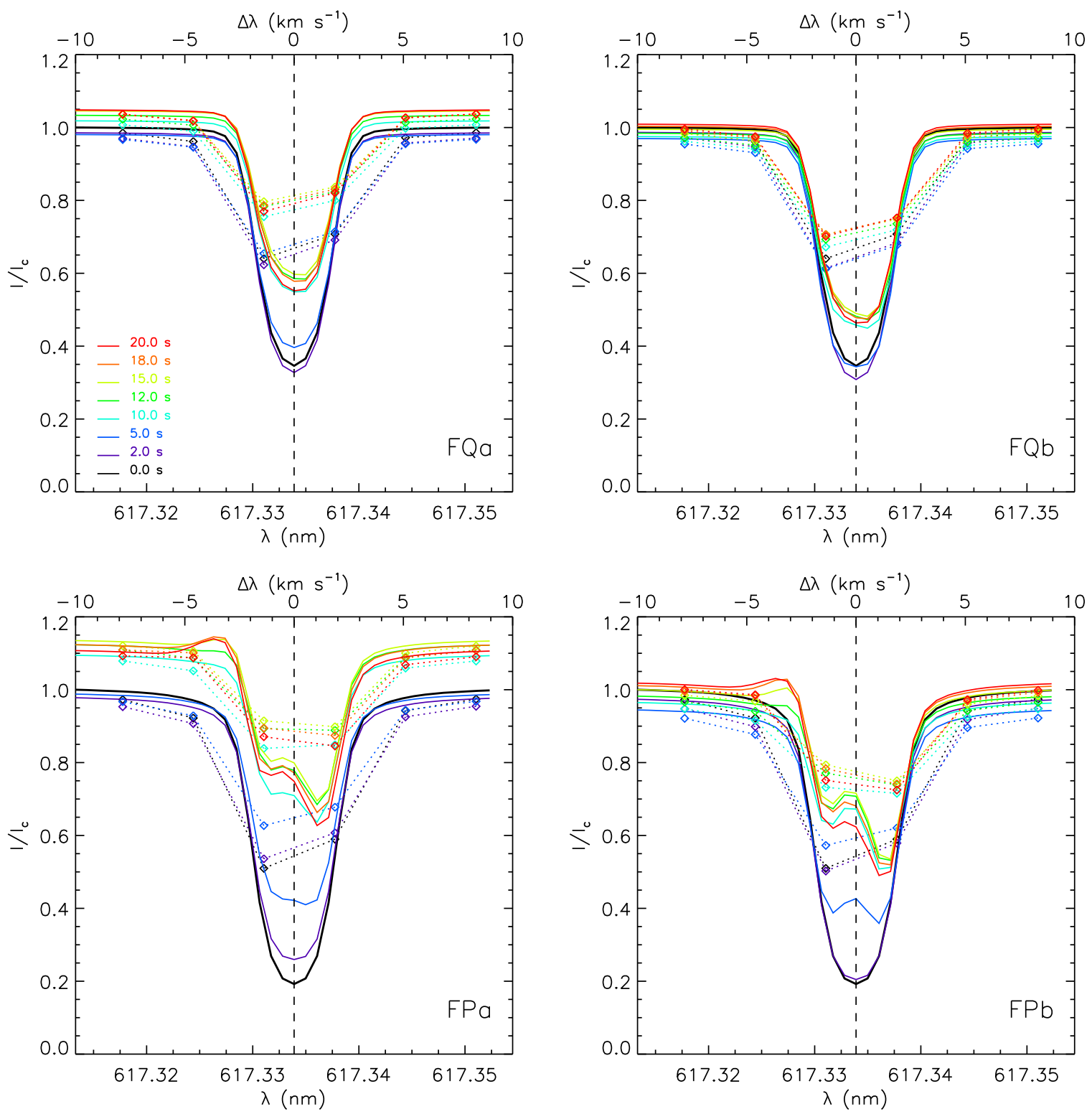

Figure 2. Time evolution of the Fe I $6173 \AA$ line profiles in the four simulation cases. The horizontal axis above marks the wavelength in Doppler velocity units, with negative sign for blueshift and positive sign for redshift. Dashed vertical lines denote the line center wavelength. Synthetic line profiles are shown in solid lines, while the simulated HMI profiles are shown as diamonds representing the observed values with the six tunable filters.

although with a lower spectral resolution, the simulated HMI profiles still show a clear intensity increase at the line center due to flare heating. However, as the positions of the six wavelength points are not symmetric to the line center, the weak blue asymmetry, found in some original profiles as mentioned above, is not clearly seen in the simulated HMI profiles. Instead, there appears a fake red asymmetry in the simulated HMI profiles for the quiet-Sun cases considered here.

\subsection{Penumbral Atmosphere}

Compared to the quiet Sun, the penumbra has a lower temperature in the lower atmosphere, but the temperature enhancement could be larger if subjected to the same electron beam (Figure 4). For Cases FPa and FPb, we do not see any dimming in the line, but rather a dimming in the continuum (Figure 2). The nonthermal electrons penetrate deeper in the atmosphere than in the quiet-Sun case. One can see a complex profile of the contribution function with two components. At $5 \mathrm{~s}$ of Case $\mathrm{FPa}$, the enhancement at the line center is still mainly caused by the backwarming effect at the line formation height, although there exists another small contribution from the beam heating. However, at the same time of Case FPb, the beam heating above the line formation height already plays a major role. In the latter case, the line source function in the chromosphere is greatly enhanced by the electron beam heating. As a result, the place where the contribution function reaches its maximum is higher than the height of optical depth unity. The line center is much more enhanced in the penumbral cases than in the quiet-Sun cases. Although the line profiles are generally in absorption, there appear an emission peak at the line center. However, the emission peak is not visible in the HMI profiles owing to the low spectral resolution of HMI, but the intensity enhancement at the line core is still larger compared with the quiet-Sun cases. We also notice a blue asymmetry in the original calculated line profile, shown as an 

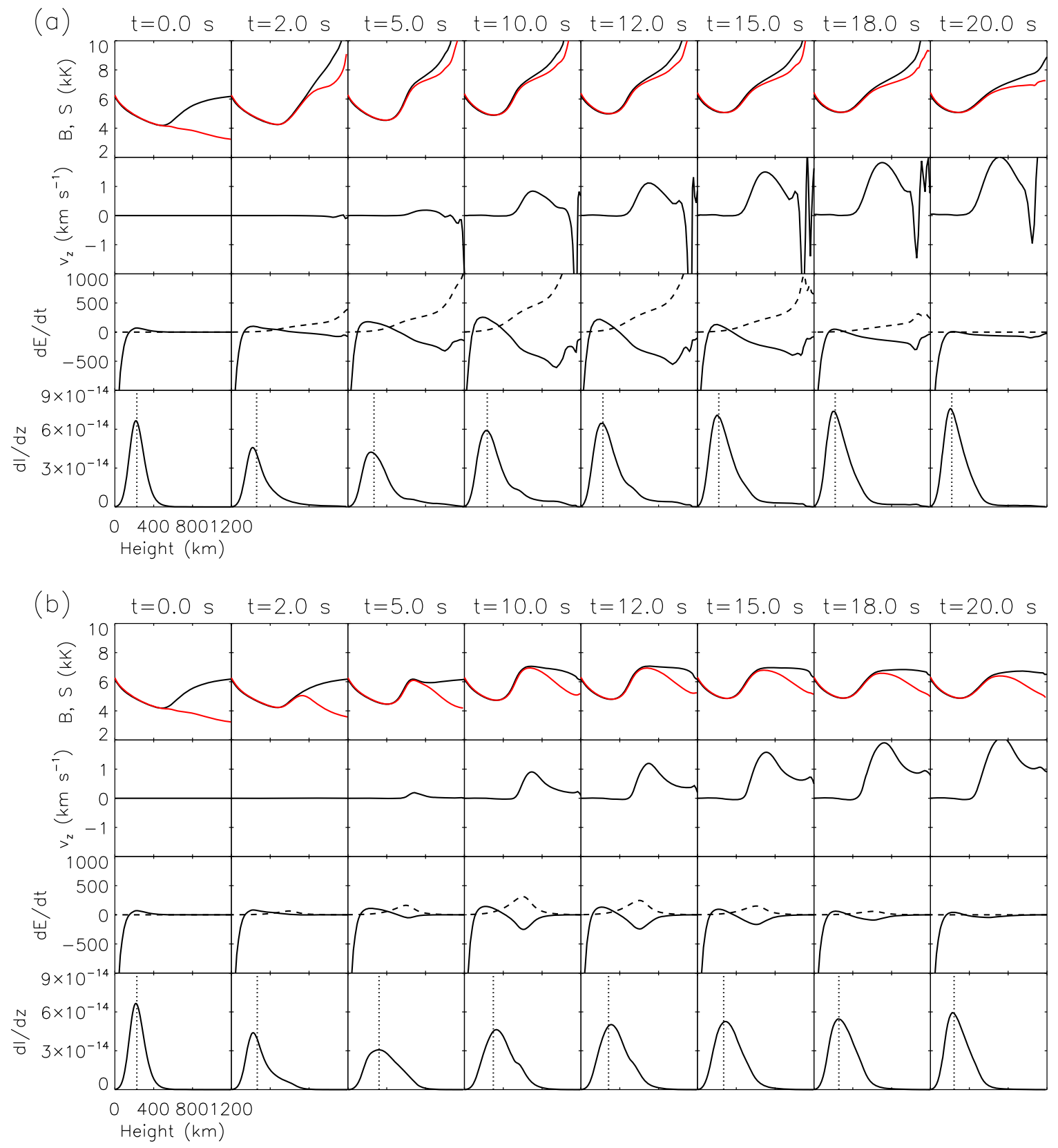

Figure 3. Time evolution of the Planck function and the line source function of Fe I $6173 \AA$, the vertical velocity, the heating rates, and the contribution function to the line intensity in Cases FQa (a) and FQb (b). In the top row of each panel, the Planck function (black) and the line source function (red) are plotted as an "equivalent" temperature. In the second row of each panel, a positive velocity means an upflow, while a negative one means a downflow, which is opposite to the definition in Figure 2. In the third row of each panel, the solid curve shows the radiative heating rate and the dashed curve shows the electron beam heating rate, with units of $\mathrm{erg} \mathrm{cm} \mathrm{cm}^{-3} \mathrm{~s}^{-1}$. In the last row of each panel, the contribution function is shown as the solid curve, while the dotted line denotes the height where the optical depth at the line center reaches unity.

enhanced blue wing and a weak blueshift of the central emission peak. The blue asymmetry comes from the mass motion in the layers of around $500 \mathrm{~km}$, where the contribution function is still large, although these layers are well above the height of optical depth unity. In the simulated HMI profiles, a fake red asymmetry appears at the beginning as in the quietSun cases, and it then gradually turns to a blue asymmetry.

\subsection{Polarized Line Profiles}

We have also modeled the Stokes line profiles using the RH code, assuming a vertical magnetic field with an exponential distribution $B(z)=B_{0} \exp (-z / H)$. The quantity $B_{0}$ is the magnetic field strength at the bottom of the photosphere, which is set to $100 \mathrm{G}$ for the quiet Sun (cases FQa and FQb), and $1000 \mathrm{G}$ for the penumbra (cases FPa and FPb). The scale height $H$ is specifically set so that the magnetic field would reduce to half of $B_{0}$ at $200 \mathrm{~km}$. The results are shown in Figure 5.

In cases $\mathrm{FQa}$ and $\mathrm{FQb}$, because the magnetic field is relatively weak, the Stokes $I$ profiles are nearly identical to the line profiles in Figure 2. One can see an increase at the line center and a weak blue asymmetry. However, in cases FPa and $\mathrm{FPb}$, there is a large difference in the Stokes $I$ profiles due to the presence of a strong magnetic field. The line width is increased here as a result of Zeeman effect. The intensity at the 

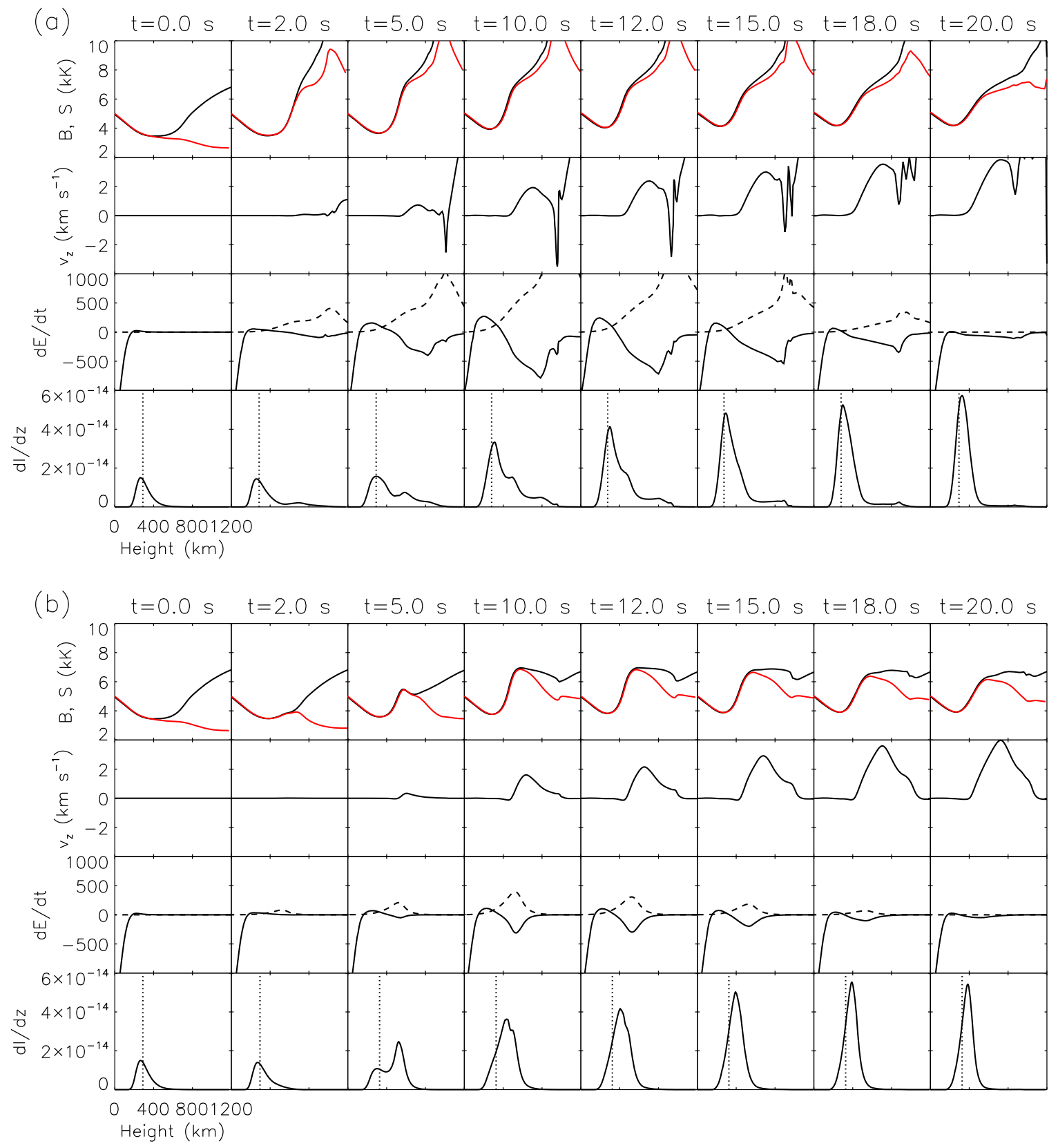

Figure 4. Same as Figure 3, but for Cases FPa (a) and FPb (b).

line center is also largely increased, but no emission peak is shown here as in Figure 2. Yet there exist two small humps at the near wings (about $\pm 0.3 \AA$ ), with a larger one at the blue wing. The simulated HMI Stokes $I$ profiles have a similar shape to those in Figure 2. For the simulated Stokes $V$ profiles, we only find a decrease in the amplitude of the lobes, but no polarity reversal, in all the simulation cases. We note that, as the HMI inversion code can automatically take into account the convolution effect by the filters, such a decrease in the magnitude of Stokes profiles does not imply a significant underestimation of the real magnetic field strength.

\section{Discussion and Summary}

In all four cases of flare simulations, we find a change of the Fe I $6173 \AA$ line in response to flare heating. Our calculations indicate that radiative backwarming and direct electron beam heating provide energy input in the photosphere and lower chromosphere, respectively; both contribute to the increase of the intensity at the line center. A higher low-energy cutoff of nonthermal electrons can increase the contribution of beam heating to the lower atmosphere and thus the line intensity. If the initial atmosphere is the quiet-Sun atmosphere, the line profile is still in absorption, but with a significant intensity increase at the line center when flare heating sets in. The formation height of the line center is still in the photosphere (around 200-300 km), but the lower chromosphere can also have some contribution to the line intensity. However, if the initial atmosphere is a cooler one, like the sunspot penumbra, the temperature enhancement is larger and the line change is more obvious under the same heating condition. The line center shows an emission peak as a result of heating in the lower atmosphere. The line source function can be increased so that the contribution function peaks above the layer of optical depth 

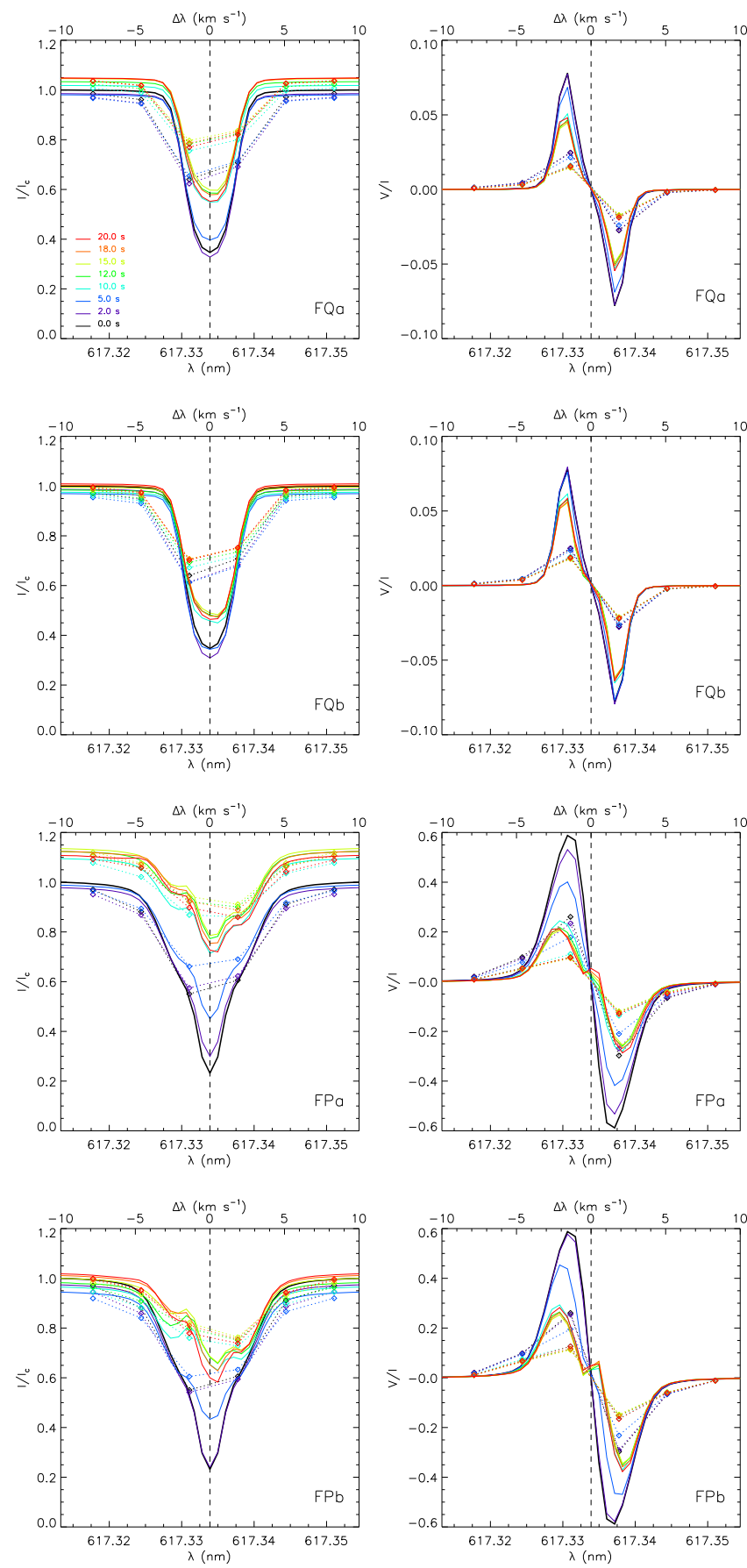

Figure 5. Time evolution of the synthetic Fe I $6173 \AA$ Stokes $I$ (left column) and $V$ (right column) profiles in the four simulation cases. Also shown are the simulated HMI profiles with the six tunable filters. The notations are the same as in Figure 2.

unity (the line formation height before flare heating). A blue asymmetry is seen as a result of mass motions in the chromosphere.

The simulated Stokes $I$ and $V$ profiles can also be altered as a result of flare heating. There is a clear intensity increase at the line center of Stokes I profiles, and the amplitude of the lobes of the Stokes $V$ profiles is greatly reduced, thus influencing the inverted magnetic fields. Considering that the flare heating, in particular by electron beam bombardment as assumed in our simulations, is impulsive and always has a short duration, our results provide a possible explanation that at least some, if not all, of the magnetic transients observed in solar flares are flareinduced artifacts. It should be noticed that some previous observations of magnetic transients associated with flares showed similar enhancement and blue asymmetry of this line (Sun et al. 2017), and occasionally an emission peak in the line center (Maurya et al. 2012; Mravcová \& Švanda 2017). Yet we would like to point out that the HMI spectral resolution can attenuate the intensity enhancement at the line center. For example, the emission peak at the line center in cases FPa and $\mathrm{FPb}$ is smoothed out and no longer visible in the simulated HMI profiles. One should also be cautious when interpreting the line asymmetries from the HMI observations, as the observed profiles would indicate a fake red asymmetry, even when the original profile is symmetric.

There have been previous calculations of the line profiles. Harker \& Pevtsov (2013) used semi-empirical models and reproduced this line in different emission stages, confirming that the spectral line profile can be altered by different temperature structures in the atmosphere. The role of beam heating in a flare is also investigated by calculations of this line from RADYN modeling results, which showed an enhancement at the line center (Sharykin et al. 2017). They used a larger spectral index $(\delta=4)$ of the electron beam and found that this line showed a redshift coinciding with the beam heating function, which is followed by a blueshift. In our results, we find the line to be generally enhanced, with an emission in the line center if the initial background atmosphere is relatively cooler like the penumbra. We only find blueshifted lines during the whole simulation time.

The fact that the response of the line to flare heating is more significant in a cooler atmosphere implies that magnetic transients should be more frequently observed in sunspot regions, which seems to be supported by observations (Kosovichev \& Zharkova 2001; Maurya et al. 2012; Burtseva et al. 2015). However, by adopting a medium-strength electron beam, we are unable to generate a full emission profile as in Harker \& Pevtsov (2013), which might be possible if we increase the energy flux of the electron beam to an exceptionally high one, as implied in some recent observations (Kowalski et al. 2017). In this way, there might also be a polarity reversal in the Stokes $V$ profiles as suggested by Harker \& Pevtsov (2013).

We thank the referee for valuable suggestions that helped improve the Letter. J.H. would like to thank Zhen Li and Yang Guo for their help in parallel computing, and Viacheslav Sadykov and Jianxia Cheng for their help in numerical calculations, and Brian Harker for providing the HMI transmission profiles. We also thank Yang Liu and Alberto Sainz Dalda for helpful discussions on the magnetic field inversion from HMI data. This work was supported by NSFC under grants 11733003 and 11533005, and NKBRSF under grant 2014CB744203, and by the Research Council of Norway through its Centres of Excellence scheme, project No. 262622. Y.L. is supported by CAS Pioneer Hundred Talents Program and XDA15052200.

\section{ORCID iDs}

Jie Hong (iD https://orcid.org/0000-0002-8002-7785

M. D. Ding (1) https://orcid.org/0000-0002-4978-4972

Ying Li (i) https://orcid.org/0000-0002-8258-4892

Mats Carlsson (iD https://orcid.org/0000-0001-9218-3139 


\section{References}

Abbett, W. P., \& Hawley, S. L. 1999, ApJ, 521, 906

Allred, J. C., Hawley, S. L., Abbett, W. P., \& Carlsson, M. 2005, ApJ, 630, 573

Allred, J. C., Hawley, S. L., Abbett, W. P., \& Carlsson, M. 2006, ApJ, 644, 484

Allred, J. C., Kowalski, A. F., \& Carlsson, M. 2015, ApJ, 809, 104

Bello González, N., Yelles Chaouche, L., Okunev, O., \& Kneer, F. 2009, A\&A, 494, 1091

Burtseva, O., Martínez-Oliveros, J. C., Petrie, G. J. D., \& Pevtsov, A. A. 2015, ApJ, 806, 173

Carlsson, M., \& Stein, R. F. 1992, ApJL, 397, L59

Carlsson, M., \& Stein, R. F. 1995, ApJL, 440, L29

Carlsson, M., \& Stein, R. F. 1997, ApJ, 481, 500

Carlsson, M., \& Stein, R. F. 2002, ApJ, 572, 626

Ding, M. D., \& Fang, C. 1989, A\&A, 225, 204

Ding, M. D., Qiu, J., \& Wang, H. 2002, ApJL, 576, L83

Dorfi, E. A., \& Drury, L. O. 1987, JCoPh, 69, 175

Edelman, F., Hill, F., Howe, R., \& Komm, R. 2004, in Proc. of SOHO 14/GONG 2004 Workshop, Helio- and Asteroseismology: Towards a Golden Future (ESA SP-559), ed. D. Danesy (Garching: ESA), 416

Gustafsson, B. 1973, UppAn, 5, 1

Harker, B. J., \& Pevtsov, A. A. 2013, ApJ, 778, 175
Hong, J., Carlsson, M., \& Ding, M. D. 2017, ApJ, 845, 144

Kosovichev, A. G., \& Zharkova, V. V. 2001, ApJL, 550, L105

Kowalski, A. F., Allred, J. C., Daw, A., Cauzzi, G., \& Carlsson, M. 2017, ApJ, 836, 12

Maurya, R. A., \& Ambastha, A. 2009, SoPh, 258, 31

Maurya, R. A., Vemareddy, P., \& Ambastha, A. 2012, ApJ, 747, 134

McTiernan, J. M., \& Petrosian, V. 1990, ApJ, 359, 524

Mravcová, L., \& Švanda, M. 2017, NewA, 57, 14

Norton, A. A., Graham, J. P., Ulrich, R. K., et al. 2006, SoPh, 239, 69

Patterson, A. 1984, ApJ, 280, 884

Patterson, A., \& Zirin, H. 1981, ApJL, 243, L99

Pereira, T. M. D., \& Uitenbroek, H. 2015, A\&A, 574, A3

Qiu, J., \& Gary, D. E. 2003, ApJ, 599, 615

Scherrer, P. H., Bogart, R. S., Bush, R. I., et al. 1995, SoPh, 162, 129

Schou, J., Scherrer, P. H., Bush, R. I., et al. 2012, SoPh, 275, 229

Sharykin, I. N., Kosovichev, A. G., Sadykov, V. M., Zimovets, I. V., \& Myshyakov, I. I. 2017, ApJ, 843, 67

Sun, X., Hoeksema, J. T., Liu, Y., Kazachenko, M., \& Chen, R. 2017, ApJ, 839,67

Uitenbroek, H. 2001, ApJ, 557, 389

Vernazza, J. E., Avrett, E. H., \& Loeser, R. 1981, ApJS, 45, 635

Xu, Y., Cao, W., Liu, C., et al. 2006, ApJ, 641, 1210 